\title{
Neural correlate of reduced respiratory chemosensitivity during chronic epilepsy
}

\section{Amol Bhandare and Nicholas Dale.}

School of Life Sciences, University of Warwick, Coventry, UK.

\begin{abstract}
While central autonomic cardiorespiratory dysfunction underlies sudden unexpected death in epilepsy (SUDEP), the specific neural mechanisms that lead to SUDEP remain to be determined. Here we took an advantage of single cell neuronal $\mathrm{Ca}^{2+}$ imaging and intrahippocampal kainic acid (KA)-induced chronic epilepsy in mice to investigate progressive changes in key cardiorespiratory brainstem circuits during chronic epilepsy. Following induction of status epilepticus (SE), we observed that the adaptive ventilatory responses to hypercapnia were reduced in mice with chronic epilepsy for 5 weeks post-SE. These changes were paralleled by reduced chemosensitivity of neurons in the retrotrapezoid nucleus (RTN), an important centre for respiratory chemosensitivity. Over the same period, chemosensory responses of the presympathetic RVLM neurons showed a slower decrease. Mice with chronic epilepsy were more sensitive to chemoconvulsants and exhibited a greatly reduced latency to seizure induction compared to naïve mice. This enhanced sensitivity to seizures, which invade the RTN, puts the chemosensory circuits at further risk and increases the chances of terminal apnoea. Our findings establish a dysfunctional breathing phenotype with its RTN neuronal correlate in mice with chronic epilepsy and suggests a functional non-invasive biomarker test, based on respiratory chemosensitivity, to identify people with epilepsy at risk of SUDEP.
\end{abstract}




\section{Introduction}

Every year 0.4-2/1,000 of people with epilepsy and 4-9/1,000 of people with drug-resistant epilepsy die as a result of sudden unexpected death in epilepsy (SUDEP)1,2. There is currently no treatment or biomarker test available to identify people that are at higher risk of SUDEP. Although studies show that SUDEP mainly occurs in people with drug-resistant and generalised tonic-clonic seizures ${ }^{1,2}$, it is still unclear why these seizures are more fatal in people with a history of seizures.

Central autonomic cardiorespiratory brainstem circuits generate the rhythms for breathing and the sympathetic and parasympathetic activity that controls the heart ${ }^{3,4}$. Evidence from epilepsy monitoring units ${ }^{1,2,5,6}$ and in vivo rodent findings ${ }^{7,8}$ implies that centrally mediated apnoea and bradycardia occurring immediately after seizures leads to SUDEP. The origins of SUDEP are therefore likely to lie in the dysfunction of these key brainstem circuits. In genetic mouse models of epilepsy, seizure-induced spreading depolarisation (recorded in the dorsal medulla) can silence the brainstem respiratory neuronal network and this has been proposed to cause SUDEP ${ }^{7,8}$. Seizure-induced spreading depolarisation was recorded as an extracellular field potential from the whole medulla in anaesthetized mice ${ }^{7}$. While this suggests disruption of key cardiorespiratory outputs from the brainstem, it does not give insight into the specific neuronal circuitry and mechanisms involved. Furthermore, until now recordings have been performed under anaesthesia, which is well known to depress neuroglial activity and thus enhance susceptibility to spreading depolarization.

A recent study has shown reduced ventilatory responses to hypercapnic challenges up to 30 days after pilocarpine-induced status epilepsy (SE) in rats ${ }^{9}$. The retrotrapezoid nucleus (RTN) contains neurons that have been identified as being involved in $\mathrm{CO}_{2}$ chemoreception. Cardiovascular function is controlled by excitatory rostral ventrolateral medullary (RVLM) neurons in the brainstem ${ }^{3,4}$. Here we utilized genetically-encoded $\mathrm{Ca}^{2+}$ indicators and gradient refractive index (GRIN) optic fibres to allow the direct visualization of dynamic cellular activity of deep brain cardiorespiratory neurons in awake mice at single cell resolution and identify how seizures alter the activity and responses of chemosensitive respiratory RTN and cardiovascular RVLM neurons. By recording from the same mouse before and following the establishment of SE for several weeks, we have documented a loss of chemosensitivity in RTN neurons, both in the magnitude of response and the number that respond to a hypercapnic challenge. We have found that mice with chronic epilepsy, compared to their pre-epileptic state, have reduced baseline respiratory activity (tidal 
volume, $\mathrm{V}_{\mathrm{T}}$ ) during inspiration of room air and during hypercapnic challenges with 3 and $6 \%$ of $\mathrm{CO}_{2}$. We propose that the reduced chemosensitivity of RTN neurons, is likely to make the central respiratory system more vulnerable to subsequent chronic spontaneous seizures, and could contribute to SUDEP.

\section{Results}

\section{Seizures spread into the central autonomic cardiorespiratory network.}

Using methods that we have previously developed, neurons of the RTN and RVLM were transduced with an AAV to drive expression of calcium reporter GCaMP6 under the control of the Syn promoter and allow assessment of their activity via a head mounted minimicroscope (Fig 1A). We prepared 15 mice (6 with injection of GCaMP6 into the RTN and 9 with injection into the RVLM, Fig 1B) and assessed their responses to hypercapnia before and after induction of SE (during chronic phase of epilepsy; 3, 5 and 7-weeks post-SE). After exclusion of 3 RTN-injected and 2 RVLM-injected mice (Fig 1B) for insufficient fluorescence signals, we analysed the remaining mice for their neuronal responses before the induction of SE and at 3, 5 and 7-weeks following SE induction with intrahippocampal kainic acid (KA) injection (Fig 1C). Neurons recorded in the RTN were identified by their position relative to the facial $\left(7^{\text {th }}\right)$ nucleus (Fig 1D) and co-staining for neuromedin B (NMB) (Fig 1E,F). Neurons in the RVLM were identified by their position relative to the nucleus ambiguous and immunoreactivity for phenylethanolamine N-methyltransferase (PNMT) (Fig 1G-I).

During seizures, recorded via EEG electrodes, large amplitude $\mathrm{Ca}^{2+}$ signals could be observed in neurons of both the RTN (Fig 2A, Supplementary Movie 1) and RVLM (Fig 2B, Supplementary Movie 2). This activity commenced around $18.9 \pm 1.5$ minutes after injection of KA (Fig 2C; there was 6 min delay between intrahippocampal injection and the start of recording) and lasted throughout the period of seizures until they were terminated by ketamine and diazepam injection. For technical reasons we did not record the EEG prior to intrahippocampal KA injection, however, as shown previously ${ }^{10-12}$, we found that the seizures had already started at 6 min after KA injection (increase in the power of the gammarange frequency $(25-45 \mathrm{~Hz})$ compared to pre-seizure EEG activity; Fig 2C).

\section{Development of a compromised breathing phenotype in mice with chronic epilepsy.}

The RTN is an important nucleus that contributes to the chemosensory control of breathing. By contrast the RVLM projects to the sympathetic and parasympathetic preganglionic neurons and controls cardiovascular parameters such as heart rate and blood pressure. 
RVLM neurons also exhibit chemosensitivity ${ }^{13}$. As seizures invade these nuclei, we expected neuronal chemosensory responses to change during the development of chronic epileptic states following induction of SE. Such changes would be likely to impact on the adaptive ventilatory responses to hypercapnia. We therefore examined how responses to hypercapnia in mice subjected to intrahippocampal KA injection or saline injection (control, surgical intervention but no SE induction) changed with time (Fig 3).

In mice with chronic epilepsy, the baseline tidal volume $\left(\mathrm{V}_{\mathrm{T}}\right)$ during breathing of room air was significantly lower when assessed 3 and 5-weeks after induction of SE (Fig $3 A$ ). This was also found for $\mathrm{V}_{\mathrm{T}}$ during mild $\left(3 \% \mathrm{CO}_{2}\right)$ and moderate $\left(6 \% \mathrm{CO}_{2}\right)$ hypercapnia (Fig $\left.3 \mathrm{~A}\right)$. Interestingly the respiratory frequency was not altered by induction of SE or during the establishment of chronic epilepsy. As might be expected, the changes in minute ventilation $\left(V_{E}\right)$ reflected the changes apparent in $V_{T}$ (Fig 3A). In control mice, $V_{T}$ in baseline (room air breathing) was increased at 7 weeks post intrahippocampal saline injection (Fig $3 B$ ). $V_{T}$ and $V_{E}$ remained stable over the entire monitoring period (Fig $3 B$ ). Thus, the changes in breathing at different levels of $\mathrm{CO}_{2}$ seen in the epileptic mice are due to the induction of epilepsy rather than the surgical intervention.

\section{Attenuated RTN chemosensitive neurons' response to hypercapnia during chronic epilepsy.}

Previously we have documented that RTN neurons exhibit a range of firing patterns during hypercapnia14: Adapting (Ad); Graded (Gr), Inhibitory (In) and non-coding (NC) (Fig 1B). As before, we found that most frequent neuronal response to hypercapnia before the induction of SE was a transient adapting pattern of activity immediately following the increase in inspired $\mathrm{CO}_{2}$ (Figs $1 \mathrm{~B}$ and 4A). At 3, 5 and 7-weeks following induction of SE, this proportion of adapting neurons (vs non-adapting subtypes) was decreased $\left(\chi^{2}(3,136)=7.86, p=0.049\right.$ ) (Fig 4A). We quantified the adapting response by averaging the individual changes in GCaMP6 fluorescence at each monitoring point (Fig 4B,C). This showed that the adapting $\mathrm{Ca}^{2+}$ signal was weaker at 3, 5 and 7-weeks following induction of SE compared to before SE. As a further quantification, we measured the area under the curve (AUC) of the adapting response during $3 \%$ inspired $\mathrm{CO}_{2}$ (Fig 4D). This again confirmed the diminished neuronal activation during hypercapnia at week 3 and 5 post-SE ( $p=0.04$ and 0.03 , respectively) $(553.9 \pm 163.0$ at pre-SE compared to $129.1 \pm 24.5,111.3 \pm 23.2$ and $138.8 \pm 33.7$ at 3, 5, and 7-weeks post-SE, respectively). 


\section{Responses of RVLM neurons to hypercapnia during chronic epilepsy.}

Neurons of the RVLM exhibited similar patterns of responses to hypercapnia as those in the RTN (Fig 1B). Like the RTN, before induction of SE the adapting response was most common. However the magnitude of these responses $\left(F / F_{0}\right)$ were less than those of the RTN neurons (compare Figs 4A,D with Figs 5A,D). Unlike the neuronal responses of the RTN, the responses to hypercapnia in RVLM neurons showed a delayed reduction of the magnitude of the adapting response relative to the pre-SE magnitude following the induction of SE that was only evident at 7 weeks post SE (Fig 5A-D, AUC for RVLM adapting responses were $164.3 \pm 21.8$ at pre-SE compared to $119.4 \pm 17.2,114.7 \pm 21.4$ and $90.3 \pm$ 14.1 at 3,5 and 7 -weeks post-SE, respectively; pre-SE vs 7 weeks post-SE $p=0.01$ ). There was also a reduction in the proportion of adapting neurons in the RVLM relative to the other types of neurons $\left(\chi^{2}(3,190)=10.4, p=0.015\right)$. The lack of an immediate strong effect of SE on the responses of RVLM neurons to hypercapnia is not because the SE was weaker in these mice as monitoring the strength of seizures (Racine scale values) during SE showed a very similar distribution for mice in which RTN and RVLM neurons were recorded (Fig 6A). In parallel with the changes to hypercapnia in RTN and RVLM neurons that occurred over the 7 weeks monitoring period, we found that the frequency of spontaneous seizures observed (in person witnessed and videotaped during regular monitoring) progressively declined until 7 weeks post-SE, before starting to rise at 9 weeks post SE (Fig 6B).

\section{Increased sensitivity and reduced latency to seizure induction in mice with chronic epilepsy.}

During SE induction through intrahippocampal injection of KA (0.3 $\mu \mathrm{g} / \mathrm{mouse})$, we recorded first seizure (visual EEG burst) with EEG electrodes at 1369.5 $\pm 302.7 \mathrm{sec}$ after KA injection (Fig 7A) and seizure lasted for $43 \pm 22 \mathrm{sec}$ (Fig 7B). During the chronic phase of epilepsy $(7.9 \pm 0.2$ weeks after induction of SE), we tested the seizure threshold in mice with 3 and 6-fold lower doses of intrahippocampal KA $(0.1 \mu \mathrm{g}$ of $\mathrm{KA} /$ mouse, $\mathrm{n}=4$ and $0.05 \mu \mathrm{g}$ of $\mathrm{KA} /$ mouse, $\mathrm{n}=7$, respectively) and compared seizure induction latency and its duration with that during the induction of SE. We found that the time for the induction of the first seizure/visual EEG burst was significantly reduced $(142.0 \pm 37.7 \mathrm{sec}$ for $0.1 \mu \mathrm{g} \mathrm{KA}$, Fig 7A, Supplementary Movie 3 (RVLM imaging) and $501.1 \pm 53.8 \mathrm{sec}$ for $0.05 \mu \mathrm{g} \mathrm{KA}$, Fig 7A, Supplementary Movie 4 (RTN imaging)) but there was no change in the duration of the resulting seizures $(47.8 \pm 13.5 \mathrm{sec}$ for $0.1 \mu \mathrm{g} \mathrm{KA}$ and $41.6 \pm 18.0 \mathrm{sec}$ for $0.05 \mu \mathrm{g} \mathrm{KA})$ (Fig 7A-B) as compared with SE. The seizures induced by the lower doses of KA were still able to invade the RTN (Fig 7C, Supplementary Movie 4) and RVLM (Fig 7D, Supplementary 
Movie 3) neurons and cause aberrant activity within these nuclei. These data suggest an increase in sensitivity and susceptibility to new seizures in mice with chronic epilepsy. As these seizures can still invade the brainstem nuclei, they are likely to further compromise the chemosensory control of breathing.

\section{Discussion}

Adaptive increases in tidal volume $\left(V_{T}\right)$ or breathing frequency $\left(f_{R}\right)$ in response to hypercapnia (chemosensory reflex) are an important component of normal central respiratory homeostasis ${ }^{3,4}$. Mice with chronic epilepsy developed a breathing phenotype with reduced $\mathrm{V}_{\mathrm{T}}$ which was evident at 3 and 5-weeks post-SE. In chronic epileptic mice baseline breathing in room air was reduced compared to the naïve state and their chemosensory adaptive responses to hypercapnia were significantly decreased at mild (3\%) and moderate $(6 \%) \mathrm{CO}_{2}$. Our findings are in line with a previous study where 30 days after induction of SE, rats showed a reduced breathing response ${ }^{9}$. Interestingly, at week-7 postSE we noted a substantial recovery of breathing phenotype in mice with chronic epilepsy possibly due to the respiratory neuronal plasticity arising from multiple cellular/synaptic mechanisms at different sites in the respiratory control system as shown before ${ }^{15,16}$.

We used miniature microscopes to image a genetically-encoded $\mathrm{Ca}^{2+}$ indicator at cellular resolution in key respiratory and cardiovascular brainstem nuclei in awake mice before, during and after the establishment of the chronic epileptic state. This avoided one of the major drawbacks of prior studies: the use of anaesthesia which unavoidably alters circuit and cellular brain function. Clinical and preclinical findings have suggested that forebrain seizures might spread to the medullary neuronal network and cause central cardiorespiratory arrest ${ }^{7,17}$. We now provide real-time cellular evidence for this by imaging the activity of individual neurons in the RTN and RVLM. This does indeed demonstrate that seizures spread into both nuclei and cause aberrant neuronal activity in these critical networks during and following the period of the seizure. This further strengthens the findings by Aiba and Noebels ${ }^{7}$ by establishing the effect of seizures on specific subsets of brainstem cardiorespiratory autonomic neurons.

In addition to the ability of seizures to invade acutely into the RTN and RVLM nuclei, we found that the chronic epileptic state caused long-lasting alterations in the ability of RTN neurons to respond to hypercapnia. Adapting responses to hypercapnia evident in the naïve state (before induction of SE) were greatly attenuated when tested 3 and 5 weeks following 
establishment of the epileptic state, with some evidence of partial recovery at 7 weeks. This provides a neural substrate that can at least partly explain why the breathing responses to hypercapnia were also reduced in the chronic epileptic state.

One third of people with epilepsy do not respond to currently available anti-epileptic medications and are more vulnerable to SUDEP. Furthermore, the incidence of SUDEP is higher in people with drug-resistant epilepsy ${ }^{1,2}$. Case studies have found that the poor seizure control, particularly of generalised tonic-clonic seizures ${ }^{18}$, a history of any seizure in last three months before SUDEP (compared to none) ${ }^{19}$ and increased frequency of tonicclonic seizures per year ${ }^{20}$ are significant risk factors for SUDEP.

Although findings from human SUDEP $\operatorname{cases}^{17,21}$ and animal studies ${ }^{7,22}$ establish a central role for dysfunctional cardiorespiratory neuronal networks as the cause of SUDEP, the specific neuronal circuits involved, and the reasons for why their dysfunction leads to cessation of breathing or cardiovascular activity are unknown. This makes prevention and treatment of SUDEP more difficult. Modelling of SUDEP is experimentally challenging due to its unpredictable nature. While we have not directly modelled SUDEP, we microinjected a chemoconvulsant into a restricted brain region to induce localised acute and chronic spontaneous seizures. This approach allowed us to discern the effects of seizures on the central cardiorespiratory system that could contribute to SUDEP. Our longitudinal imaging of neurons pre- and post-SE induction enabled us to document the effects of repeated chronic spontaneous seizures, a major risk factor for SUDEP, on the function of cardiorespiratory neurons and hypercapnic breathing responses. The weakening of the chemosensory reflex via loss of chemosensitivity in the RTN is likely to increase the risk of SUDEP. For example, we speculate that if breathing were to cease due to invasion of spreading depression into the brainstem, arterial blood would become hyercapnic and acidic. Ordinarily, this would act as a powerful stimulant to restart breathing once the spreading depression had passed. However, the weakened chemosensory responses of RTN neurons that we observed in the chronic epileptic state are likely to make it harder to restart breathing and could thus increase the risk of SUDEP. This speculation needs to be tested in a more specific animal model of SUDEP, e.g. DBA/1 mice where the incidence of respiratory arrest following audiogenic seizures increases with seizure repetition ${ }^{23}$.

While we have not studied the effect of seizures on chemosensory neurons of the raphe nucleus, agonists for serotonin receptors (a key raphe neurotransmitter) have a protective 
effect in blocking seizure-induced SUDEP in DBA mice ${ }^{24}$ and mice with genetic deletion of serotonin neurones ${ }^{25}$. Raphe neurons project to the RTN and contribute to chemosensory responses in this nucleus ${ }^{26}$. Thus, activation of serotonin receptors could help to compensate for the weakened responses of the chemosensitive RTN neurons and enhance the stimulatory effect of $\mathrm{CO}_{2}$ on breathing ${ }^{26,27}$. Nevertheless, the action of serotonin could also be mediated through its direct effect on seizure activity ${ }^{25,28}$, arousal ${ }^{29}$, sleep-wake regulation and circadian rhythm ${ }^{30}$ and warrants further investigation.

In contrast to altered RTN neuronal responses to hypercapnia during chronic epilepsy, RVLM neurons exhibited a much slower reduction in their adaptive responses that only became statistically significant at 7 weeks after SE. Findings from epilepsy monitoring units $^{2,6,17,21,31}$ and preclinical studies $8,9,23$ indicate that respiratory rather than cardiac failure is the major contributor to SUDEP. Our findings are thus consistent with this, as they show a greater immediate effect of repeated seizures on the RTN chemosensory neurons than on the RVLM neurons. Although, the central respiratory apnoea contributes to SUDEP, it sets in train a sequence of events that include hypoxemia and acidosis that could indirectly trigger failure of the central cardiovascular system and eventual cardiac arrest.

A final implication of our findings is that longitudinal monitoring of respiratory chemosensitivity, combined with other established clinical risk factors, could be a useful biomarker test to identify people at risk of SUDEP. Although a pre-epileptic baseline for respiratory chemosensitivity could not be established in people with epilepsy, regular measurement of chemosensitivity via a simple non-invasive breathing test could establish trends in chemo-responsiveness that could indicate increased or diminished risk of SUDEP.

\section{Materials and Methods}

Experiments were performed in accordance with the European Commission Directive 2010/63/EU (European Convention for the Protection of Vertebrate Animals used for Experimental and Other Scientific Purposes) and the United Kingdom Home Office (Scientific Procedures) Act (1986) with project approval from the University of Warwick's AWERB (PP1674884).

\section{Viral handling}

We used AAV-9-syn-GCaMP6s vector with synapsin promoter (pGP-AAV-syn-GCaMP6sWPRE.4.641 at a titre of $1 \times 10^{13} \mathrm{GC} \cdot \mathrm{ml}^{-1}$, Addgene, Watertown, MA, USA), and therefore it 
transduced neurons showing higher tropism for the AAV 2/9 subtype, and did not transduce non-neuronal cells. The AAV uses the synapsin promoter. Virus was aliquoted and stored at $-80^{\circ} \mathrm{C}$. On the day of injection, it was removed and stored at $4{ }^{\circ} \mathrm{C}$, loaded into graduated glass pipettes (Drummond Scientific Company, Broomall, PA, USA), and placed into an electrode holder for pressure injection.

\section{Viral transfection of RVLM and RTN neurons}

Adult male C57BL/6J mice (8-10 weeks old and 20-30 g) were anaesthetized with isofluorane (4\%; Piramal Healthcare Ltd, Mumbai, India) in pure oxygen (4 L· $\left.\mathrm{min}^{-1}\right)$. Adequate anaesthesia was maintained with $0.5-2 \%$ Isofluorane in pure oxygen $\left(1 \mathrm{~L} \cdot \mathrm{min}^{-1}\right)$ throughout the surgery. Mice received a presurgical subcutaneous injection of atropine (120 $\mu \mathrm{g} \cdot \mathrm{kg}^{-1}$; Westward Pharmaceutical Co., Eatontown, NJ, USA) and meloxicam (2 mg $\mathrm{kg}^{-1}$; Norbrook Inc., Lenexa, KS, USA). Mice were placed in a prone position into a digital stereotaxic apparatus (Kopf Instruments, Tujunga, CA, USA) on a heating pad (TCAT 2-LV: Physitemp, Clifton, NJ, USA) and body temperature was maintained at a minimum of $33^{\circ} \mathrm{C}$ via a thermocouple. The head was levelled, at bregma and $2 \mathrm{~mm}$ caudal to bregma, and graduated glass pipettes containing the virus were placed stereotaxically into either the RTN or RVLM (Fig 1D,G). The RTN was defined as the area ventral to the caudal half of the facial nucleus, bound medially and laterally by the edges of the facial nucleus (coordinates with a $9^{0}$ injection arm angle: $-1.0 \mathrm{~mm}$ lateral and $-5.6 \mathrm{~mm}$ caudal from Bregma, and -5.2 to $-5.5 \mathrm{~mm}$ ventral from the surface of the cerebellum; Fig 1D). The RVLM defined as a triangular area ventral to the nucleus ambiguous (NA), lateral to the inferior olive (IO) or pyramidals (Py) and medial to the spinal trigeminal sensory nucleus (Sp5) (coordinates with a $0^{\circ}$ injection arm angle: $1.3 \mathrm{~mm}$ lateral and $-5.7 \mathrm{~mm}$ caudal from Bregma, and $-5.15 \mathrm{~mm}$ ventral from the surface of the cerebellum; Fig 1G). The virus solution was pressure injected $(<300 \mathrm{~nL})$ unilaterally. Pipettes were left in place for 3-5 minutes to prevent back flow of the virus solution up the pipette track. Postoperatively, mice received intraperitoneal (IP) injections of buprenorphine (100 $\mu \mathrm{g} \cdot \mathrm{kg}^{-1}$; Reckitt Benckiser, Slough, UK). Mice were allowed 2 weeks for recovery and viral expression, with food and water ad libitum.

\section{Implantation of EEG electrodes, hippocampal cannula and GRIN lens}

Mice expressing GCaMP6 were anaesthetized with isofluorane, given pre-surgical drugs, placed into a stereotax, and the head was levelled as described above. Four holes were drilled for implantation of 2 EEG recording, a ground and a reference electrode. EEG electrodes were PFA-coated silver wire of diameter: $0.254 \mathrm{~mm}$ (Bilaney Consultants Ltd, UK) 
(coordinates with a $0^{\circ}$ injection arm angle: Recording electrodes- $\pm 1.5 \mathrm{~mm}$ lateral and 1.0 $\mathrm{mm}$ rostral from Bregma, ground and reference electrodes- $\pm 2.5 \mathrm{~mm}$ lateral and $-1.5 \mathrm{~mm}$ caudal from Bregma). Silver wires were implanted and secured in position with SuperBond ${ }^{\mathrm{TM}}$ (Prestige Dental, Bradford, UK). EEG wires were passed through the pedestal (Bilaney Consultants Ltd, UK), and pedestal was implanted and secured in place over the head with SuperBond ${ }^{\mathrm{TM}}$. Another two holes were drilled for implantation of hippocampal cannula (Fig $1 \mathrm{C}$, coordinates with a $0^{\circ}$ injection arm angle: $1.8 \mathrm{~mm}$ lateral and $-2.0 \mathrm{~mm}$ caudal from Bregma, and $-1.6 \mathrm{~mm}$ ventral from the surface of the cerebellum) and GRIN lens (coordinates as above). Stainless steel cannula, 26 gauge and $10 \mathrm{~mm}$ long, was implanted in the hippocampus and secured in place with SuperBond ${ }^{\mathrm{TM}}$. To widen the lens path whilst producing the least amount of deformation of tissue, a graduated approach was taken; firstly a glass pipette was inserted down the GRIN lens path to a depth $500 \mu \mathrm{m}$ above where the lens would terminated and left in place for 3 mins; this procedure was then repeated with a blunted hypodermic needle. The GRIN lens (600 $\mu \mathrm{m}$ diameter, $7.3 \mathrm{~mm}$ length; Inscopix, Palo Alto, CA, USA) was then slowly inserted at a rate of $100 \mu \mathrm{m} \cdot \mathrm{min}^{-1}$ to a depth $\sim 1300 \mu \mathrm{m}$ above the target site, then lowered at a rate of $50 \mu \mathrm{m} \cdot \mathrm{min}^{-1}$ to a depth $\sim 300 \mu \mathrm{m}$ above the RTN or RVLM target region (coordinates as above with $300 \mu \mathrm{m}$ above the target site). The lens was then secured in place with SuperBond ${ }^{\mathrm{TM}}$. Postoperatively, mice received buprenorphine, and were allowed 2 weeks for recovery, with food and water ad libitum.

\section{Baseplate installation}

Mice expressing GCaMP6 and implanted with EEG electrodes, hippocampal cannula and GRIN lens were anaesthetized with isofluorane, given pre-surgical drugs, and placed into a stereotax as described above. To hold the miniaturized microscope during recordings, a baseplate was positioned over the lens and adjusted until the cells under the GRIN lens were in focus. The baseplate was then secured with superbond ${ }^{\mathrm{TM}}$, and coated in black dental cement (Vertex Dental, Soesterberg, the Netherlands) to stop interference of the recording from ambient light. Mice were allowed 1 week for recovery, with food and water ad libitum.

Induction of status epilepsy (SE) with EEG recording and $\mathrm{Ca}^{2+}$ imaging in freely moving mice All mice were trained with dummy camera and habituated to plethysmography and flexi glass open field chamber before imaging. Instrumented mice were anaesthetized with isofluorane and placed into a stereotax as described above. Mice EEG electrodes were connected to wires and miniature microscope with integrated 475 nm LED (Inscopix, Palo Alto, CA, USA) 
was secured into the baseplate. SE was induced in mice with $0.3 \mu \mathrm{g}(50 \mathrm{nl})$ of kainic acid (KA) injected through hippocampal cannula with Hamilton Neuros syringe (Model 75 RN, Essex Scientific Laboratory Supplies Ltd., UK). Mice were taken off the anaesthesia and placed into open field chamber. The EEG recording and $\mathrm{Ca}^{2+}$ imaging were started $\sim 6$ min after KA injection. Mice were scored for their behavioural seizures using Racine scale (1Rigid posture or immobility; 2- Stiffened, extended, and often arched (Straub) tail; 3- Partial body clonus, including forelimb or hindlimb clonus or head bobbing; 3.5- Whole body continuous clonic seizures while retaining posture; 4- Rearing; 4.5- Severe whole body continuous clonic seizures while retaining posture; 5- Rearing and falling; 6- Tonic-clonic seizures with loss of posture or jumping). At 60 minutes or recording 1-2 of class 5/6 scale seizure following injection of KA (whichever happened earlier), mice were anaesthetized with isofluorane and SE was terminated with intraperitoneal injection of ketamine $(50 \mathrm{mg} / \mathrm{kg})$ and diazepam $(20 \mathrm{mg} / \mathrm{kg})$.

During SE, EEG activity was recorded, amplified and filtered using the NeuroLog system (Digitimer, Welwyn Garden City, UK) connected to a 1401 interface and acquired on a computer using Spike2 software (Cambridge Electronic Design, Cambridge, UK). The EEG activity raw data were DC removed. The power in the "gamma" frequency range (25- 45 $\mathrm{Hz}$ ) was analysed, as previously shown ${ }^{11,12}$. A power spectrum analysis of EEG (AUC, $V^{2}$ ) was done from 2 min blocks taken every 4 min apart after the start of recording. Video data was recorded with Spike2 software and was synchronised with the EEG activity (Supplementary Movie 1-2). GCaMP6 fluorescence was visualised during SE through the GRIN lens, using nVista 2 HD acquisition software (Inscopix, Palo Alto, CA, USA). Calcium fluorescence was optimised for each experiment so that the histogram range was 150-600, with average recording parameters set at 10 frames/sec with the LED power set to 10-20 $\mathrm{mW}$ of light and a digital gain of 1.0-4.0. A TTL pulse was used to synchronize the calcium signalling to the behavioural data and EEG trace. All images were processed using Inscopix data processing software (Inscopix, Palo Alto, CA, USA). GCaMP6 movies were ran through: preprocessing algorithm (with spatial and temporal downsampling), crop, spatial filter algorithm $(0.005-0.5 \mathrm{~Hz})$, motion correction and cell identification through CNMF-E (Constrained Nonnegative Matrix Factorization for microEndoscopic data) analysis to generate the identified cell sets. Cell sets were imported into Spike2 software for processing. All $\mathrm{Ca}^{2+}$ traces from specific time point were averaged and analysed for area under the curve (AUC) between the start 3\% hypercapnia and $180 \mathrm{sec}$ after it. 


\section{Plethysmography}

Mice were placed into a custom-made $0.5 \mathrm{~L}$ plethysmography chamber, with an airflow rate of $1 \mathrm{I} \cdot \mathrm{min}^{-1}$. The plethysmography chamber was heated to $31^{\circ} \mathrm{C}$ (thermoneutral for C57/BL6 mice). $\mathrm{CO}_{2}$ concentrations were sampled via a Hitech Intruments (Luton, UK) GIR250 Dual Sensor Gas analyzer or ML206 gas analyzer (ADinstruments, Sydney, Australia) connected to the inflow immediately before entering the chamber. The analyser had a delay of $\sim 15-20$ $\mathrm{sec}$ to read-out the digital output of gas mixture. Pressure transducer signals and $\mathrm{CO}_{2}$ measurements were amplified and filtered using the NeuroLog system (Digitimer, Welwyn Garden City, UK) connected to a 1401 interface and acquired on a computer using Spike2 software (Cambridge Electronic Design, Cambridge, UK). Video data was recorded with Spike2 software and was synchronised with the breathing trace. Airflow measurements were used to calculate: tidal volume $\left(\mathrm{V}_{\mathrm{T}}\right.$ : signal trough at the end of expiration subtracted from the peak signal during inspiration, converted to $\mathrm{mL}$ following calibration), and respiratory frequency ( $f R$ : breaths per minute). Minute ventilation $\left(V_{E}\right)$ was calculated as $V_{T} x f R$.

\section{Hypercapnia in freely behaving mice}

Mice were tested for hypercapnic challenge before and 3,5 and 7-weeks after induction of SE. Instrumented mice were allowed $\sim 30$ mins to acclimate to the plethysmograph. The LED was activated through a TTL pulse synchronised with the Spike2 recording and $3 \mathrm{~min}$ of baseline recordings were taken (gas mixture: $0 \% \mathrm{CO}_{2} 21 \% \mathrm{O}_{2} 79 \% \mathrm{~N}_{2}$ ). The mice were then exposed to 3 min epochs of hypercapnic gas mixture at different concentrations of $\mathrm{CO}_{2}(3$, and $6 \%$ in $21 \% \mathrm{O}_{2}$ balanced $\mathrm{N}_{2}$ ). Following exposure to the hypercapnic gas mixtures, $\mathrm{CO}_{2}$ levels were reduced back to $0 \%$ and calcium signals were recorded for a further 4 minutes recovery period.

\section{Seizure threshold in mice with chronic epilepsy}

After recording 3, 5 and 7-weeks post-SE hypercapnia responses and during chronic phase of epilepsy (8-weeks post-SE), mice were tested for seizure threshold. Instrumented mice were anaesthetized with isofluorane, placed into a stereotax and connected to EEG electrodes and miniature microscope as described above. Mice were injected through intrahippocampal cannula with 3 and 6-fold lower dose of KA $(0.1 \mu \mathrm{g}, \mathrm{n}=4$ and $0.05 \mu \mathrm{g}, \mathrm{n}=7$ of $\mathrm{KA} /$ mouse) compared to SE. Mice were taken off the anaesthesia, placed into open field chamber and EEG recording and $\mathrm{Ca}^{2+}$ imaging were started as stated above. Mice were recorded until first EEG seizure burst was recorded (Supplementary Movie 3-4) and seizures were terminated as above. 


\section{Statistical analysis}

Statistical analysis was performed in GraphPad Prism software (version 9.1.0). Statistical significance for changes in breathing was determined using two-way repeated measure ANOVA followed by $t$ tests with the Tukey's correction and shown as a violin plot. Statistical analysis of the changes in RTN adapting AUC response was via the Brown-Forsythe and Welch ANOVA with Dunnett T3 correction. This alternative test was used as the data were not normally distributed and the variance differed between groups in the comparison, thus invalidating use of a conventional ANOVA. The RVLM adapting responses and seizure delay and duration time were analysed using a one-way ANOVA followed with the Dunnett's correction. Results are shown as bar graphs with superimposed data points. Comparisons were done between pre- and post-treatment (SE or control groups). $\mathrm{P} \leq 0.05$ was considered significant. ${ }^{* * *} p \leq 0.0001,{ }^{* * *} p \leq 0.001,{ }^{* *} p \leq 0.01$ and ${ }^{*} p \leq 0.05$.

\section{Immunohistochemistry}

Mice were humanely killed by pentobarbital overdose $\left(>100 \mathrm{mg} \cdot \mathrm{kg}^{-1}\right)$ and transcardially perfused with paraformaldehyde solution (4\% PFA; Sigma-Aldrich, St Louis, MO, USA). The head was removed and postfixed in PFA $\left(4^{\circ} \mathrm{C}\right)$ for 3 days to preserve the lens tract. The brains were removed and postfixed in PFA $\left(4^{\circ} \mathrm{C}\right)$ overnight. Brainstems were serially sectioned at 50-70 $\mu \mathrm{m}$. Free-floating sections were incubated for 1 hour in a blocking solution (PBS containing $0.1 \%$ Triton $\mathrm{X}-100$ and 5\% BSA). Primary antibodies (rabbit antiNeuromedin-B [NMB; 1:1000; SAB1301059; Sigma-Aldrich, St Louis, MO, USA], or rabbit anti-phenylethanolamine N-Methyltransferase [PNMT; 1:500; 204179-T36-SIB; Stratech, Ely, UK] antibody), or (chicken anti-GFAP [GFAP; 1:500; ab4674; Abcam PLC, Cambridge, UK], or (mouse anti-NeuN [NeuN; 1:500; ab104224; Abcam PLC, Cambridge, UK] were added and tissue was incubated overnight at room temperature.

Slices were washed in PBS $(6 \times 5$ mins $)$ and then the secondary antibodies were added: either donkey anti-rabbit Alexa Fluor 680 (1:250; Jackson Laboratory, Bar Harbor, ME, USA), or donkey anti-rabbit Alexa Fluor 594 (1:250; Jackson Laboratory, Bar Harbor, ME, USA), or donkey anti-mouse Alexa Fluor 680 (1:250; Jackson Laboratory, Bar Harbor, ME, USA) antibody, or donkey anti-chicken Alexa Fluor 594 (1:250; Jackson Laboratory, Bar Harbor, ME, USA) antibody tissue was then incubated for 2-4 hours at room temperature. Tissue was washed in PBS $(6 \times 5 \mathrm{~min})$. Slices were mounted, coverslipped 
and examined using a Zeiss 880 confocal microscope with ZEN aquisition software (Zeiss, Oberkochen, Germany).

\section{Acknowledgements}

Funding: This work was supported by the Epilepsy Research UK (ERUK) Project Grant. ND was a Royal Society Wolfson Research Merit Award Holder. We thank Dr Mark Wall and Dr Robert Dallmann for their valuable feedback on the manuscript.

\section{Author contributions}

$A B$ designed the study, performed experiments, recordings, immunohistochemistry analysed data and contributed to writing of the manuscript; ND conceived and designed the study, analysed data and contributed to writing of the manuscript.

Competing interests: The authors declare that they have no competing interests.

Data and Materials availability: Raw data available on request.

\section{References:}

1 Tomson, T., Nashef, L. \& Ryvlin, P. Sudden unexpected death in epilepsy: current knowledge and future directions. Lancet Neurol. 7, 1021-1031, doi:10.1016/S1474-4422(08)70202-3 (2008).

2 Ryvlin, P. et al. Incidence and mechanisms of cardiorespiratory arrests in epilepsy monitoring units (MORTEMUS): a retrospective study. Lancet Neurol. 12, 966-977, doi:10.1016/S14744422(13)70214-X (2013).

3 Spyer, K. M. \& Gourine, A. V. Chemosensory pathways in the brainstem controlling cardiorespiratory activity. Philos. Trans. R. Soc. Lond. B Biol. Sci. 364, 2603-2610, doi:10.1098/rstb.2009.0082 (2009).

4 Guyenet, P. G. Regulation of breathing and autonomic outflows by chemoreceptors. Compr. Physiol. 4, 1511-1562, doi:10.1002/cphy.c140004 (2014).

5 Sivathamboo, S. et al. Cardiorespiratory and autonomic function in epileptic seizures: A video-EEG monitoring study. Epilepsy Behav. 111, doi:10.1016/j.yebeh.2020.107271 (2020). So, E. L., Sam, M. C. \& Lagerlund, T. L. Postictal central apnea as a cause of SUDEP: Evidence from near-SUDEP incident. Epilepsia 41, 1494-1497 (2000).

7 Aiba, I. \& Noebels, J. L. Spreading depolarization in the brainstem mediates sudden cardiorespiratory arrest in mouse SUDEP models. Sci. Transl. Med. 7, 282ra246, doi:10.1126/scitranslmed.aaa4050 (2015).

8 Jefferys, J. G. R., Arafat, M. A., Irazoqui, P. P. \& Lovick, T. A. Brainstem activity, apnea, and death during seizures induced by intrahippocampal kainic acid in anaesthetized rats. Epilepsia 60, 2346-2358, doi:10.1111/epi.16374 (2019).

9 Maia, O. A. C. et al. Pilocarpine-induced status epilepticus reduces chemosensory control of breathing. Brain Res. Bull. 161, 98-105, doi:https://doi.org/10.1016/j.brainresbull.2020.05.002 (2020). 
10 Cavalheiro, E. A., Riche, D. A. \& Le Gal La Salle, G. Long-term effects of intrahippocampal kainic acid injection in rats: A method for inducing spontaneous recurrent seizures. Electroencephalogr. Clin. Neurophysiol. 53, 581-589, doi:10.1016/0013-4694(82)90134-1 (1982).

11 Bhandare, A. M., Kapoor, K., Pilowsky, P. M. \& Farnham, M. M. Seizure-Induced Sympathoexcitation Is Caused by Activation of Glutamatergic Receptors in RVLM That Also Causes Proarrhythmogenic Changes Mediated by PACAP and Microglia in Rats. J. Neurosci. 36, 506-517, doi:10.1523/JNEUROSCI.2584-15.2016 (2016).

12 Gurbanova, A. A., Aker, R. G., Sirvanci, S., Demiralp, T. \& Onat, F. Y. Intra-amygdaloid injection of kainic acid in rats with genetic absence epilepsy: the relationship of typical absence epilepsy and temporal lobe epilepsy. J. Neurosci. 28, 7828-7836, doi:10.1523/JNEUROSCI.1097-08.2008 (2008).

13 Koganezawa, T. \& Paton, J. F. R. Intrinsic chemosensitivity of rostral ventrolateral medullary sympathetic premotor neurons in the in situ arterially perfused preparation of rats. Exp. Physiol. 99, 1453-1466, doi:10.1113/expphysiol.2014.080069 (2014).

14 Bhandare, A. et al. Analyzing the neuroglial brainstem circuits for respiratory chemosensitivity in freely moving mice. bioRxiv doi: https://doi.org/10.1101/492041, 492041, doi:10.1101/492041 (2020).

15 Ling, L. et al. Chronic Intermittent Hypoxia Elicits Serotonin-Dependent Plasticity in the Central Neural Control of Breathing. J. Neurosci. 21, 5381-5388, doi:10.1523/jneurosci.2114-05381.2001 (2001).

16 Dahan, A., Nieuwenhuijs, D. \& Teppema, L. Plasticity of central chemoreceptors: Effect of bilateral carotid body resection on central CO2 sensitivity. PLoS Med. 4, 1195-1204, doi:10.1371/journal.pmed.0040239 (2007).

17 Dlouhy, B. J. et al. Breathing Inhibited When Seizures Spread to the Amygdala and upon Amygdala Stimulation. J. Neurosci. 35, 10281-10289, doi:10.1523/JNEUROSCI.0888-15.2015 (2015).

18 Walczak, T. S. et al. Incidence and risk factors in sudden unexpected death in epilepsy: a prospective cohort study. Neurology 56, 519-525, doi:10.1212/wnl.56.4.519 (2001).

19 Langan, Y., Nashef, L. \& Sander, J. W. Case-control study of SUDEP. Neurology 64, 1131-1133, doi:10.1212/01.wnl.0000156352.61328.cb (2005).

20 Nilsson, L., Farahmand, B. Y., Persson, P. G., Thiblin, I. \& Tomson, T. Risk factors for sudden unexpected death in epilepsy: a case-control study. Lancet 353, 888-893, doi:10.1016/s0140-6736(98)05114-9 (1999).

21 Mueller, S. G., Bateman, L. M. \& Laxer, K. D. Evidence for brainstem network disruption in temporal lobe epilepsy and sudden unexplained death in epilepsy. Neuroimage Clin. 5, 208216, doi:10.1016/j.nicl.2014.06.010 (2014).

22 Bhandare, A. M. et al. Inhibition of microglial activation with minocycline at the intrathecal level attenuates sympathoexcitatory and proarrhythmogenic changes in rats with chronic temporal lobe epilepsy. Neurosci. 350, 23-38, doi:10.1016/j.neuroscience.2017.03.012 (2017).

23 Faingold, C. L., Randall, M. \& Tupal, S. DBA/1 mice exhibit chronic susceptibility to audiogenic seizures followed by sudden death associated with respiratory arrest. Epilepsy Behav. 17, 436-440, doi:10.1016/j.yebeh.2010.02.007 (2010).

24 Faingold, C. L., Randall, M., Mhaskar, Y. \& Uteshev, V. V. Differences in serotonin receptor expression in the brainstem may explain the differential ability of a serotonin agonist to block seizure-induced sudden death in DBA/2 vs. DBA/1 mice. Brain Res. 1418, 104-110, doi:10.1016/j.brainres.2011.08.043 (2011). 
25 Buchanan, G. F., Murray, N. M., Hajek, M. A. \& Richerson, G. B. Serotonin neurones have anti-convulsant effects and reduce seizure-induced mortality. J. Physiol. 592, 4395-4410, doi:10.1113/jphysiol.2014.277574 (2014).

$26 \mathrm{Wu}, \mathrm{Y}$. et al. Chemosensitivity of Phox2b-expressing retrotrapezoid neurons is mediated in part by input from 5-HT neurons. J. Physiol. 597, 2741-2766, doi:https://doi.org/10.1113/JP277052 (2019).

27 Mulkey, D. K. et al. Serotonergic Neurons Activate Chemosensitive Retrotrapezoid Nucleus Neurons by a pH-Independent Mechanism. J. Neurosci. 27, 14128-14138, doi:10.1523/jneurosci.4167-07.2007 (2007).

28 Schoonjans, A. et al. Low-dose fenfluramine significantly reduces seizure frequency in Dravet syndrome: a prospective study of a new cohort of patients. Eur. J. Neurol. 24, 309-314, doi:https://doi.org/10.1111/ene.13195 (2017).

29 Buchanan, G. F. \& Richerson, G. B. Central serotonin neurons are required for arousal to CO2. Proc. Natl. Acad. Sci. U. S. A. 107, 16354-16359, doi:10.1073/pnas.1004587107 (2010).

30 Miyamoto, H., Nakamaru-Ogiso, E., Hamada, K. \& Hensch, T. K. Serotonergic Integration of Circadian Clock and Ultradian Sleep-Wake Cycles. J. Neurosci. 32, 14794-14803, doi:10.1523/jneurosci.0793-12.2012 (2012).

31 Bateman, L. M., Spitz, M. \& Seyal, M. Ictal hypoventilation contributes to cardiac arrhythmia and SUDEP: report on two deaths in video-EEG-monitored patients. Epilepsia 51, 916-920, doi:10.1111/j.1528-1167.2009.02513.x (2010).

\section{Figures and Legends:}

A

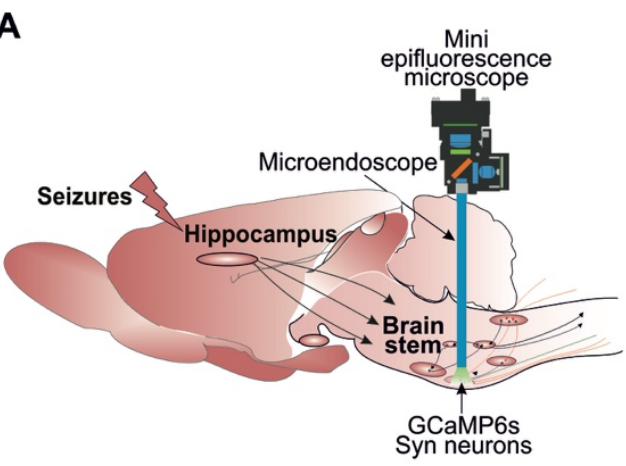

B

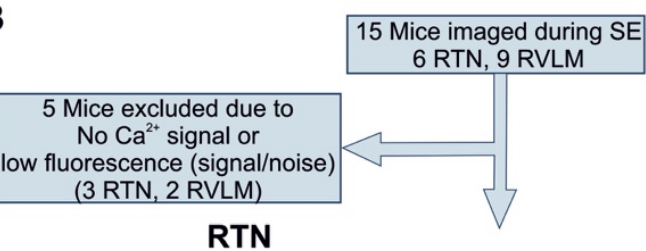

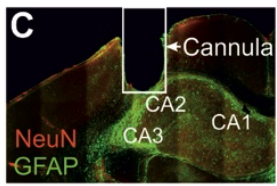

RVLM

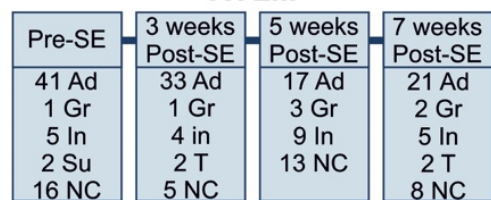

D Bregma -5.88

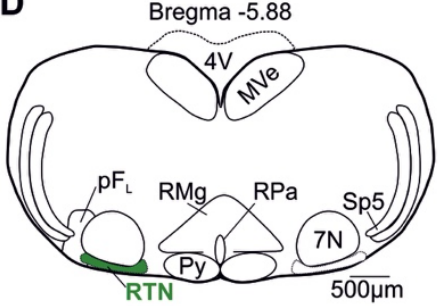

G

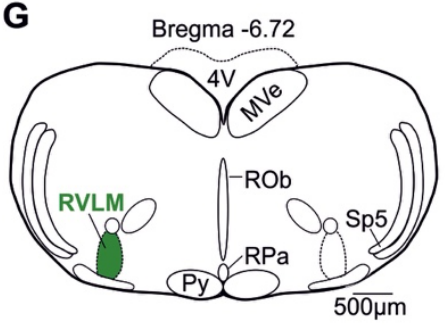

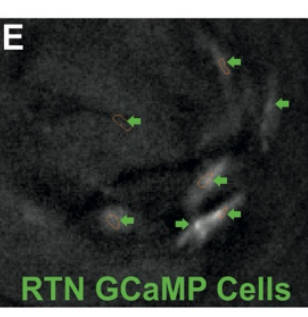

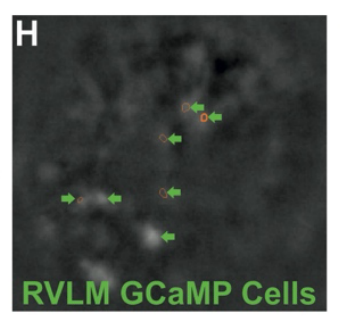

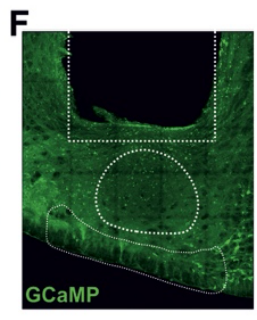
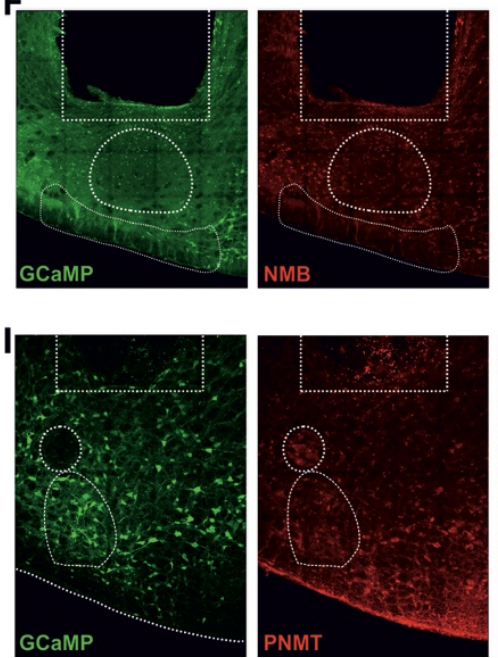
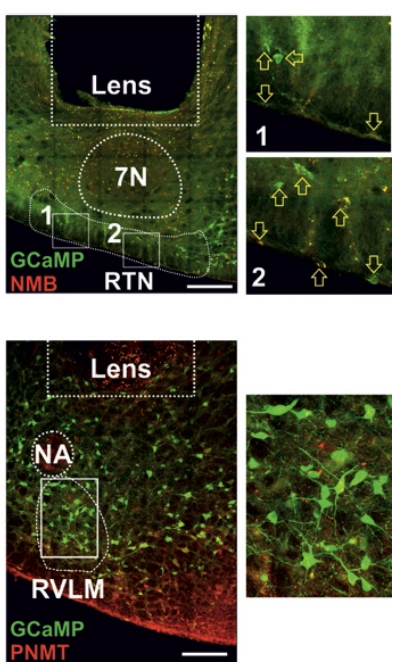

Figure 1: A) Representation of GRIN lens (microendoscope), baseplate, and mini epifluorescence camera placement for recording of brainstem nuclei with hippocampal seizure induction. B) A CONSORT flow diagram for inclusion/exclusion of experiments and 
cells classification. C) Hippocampal cannula placement for injection of KA. D) AAV9-SynGCaMP6s injection into the RTN. E) GCaMP6s fluorescence of transduced RTN neurons in freely behaving mice. F) Micrograph of lens placement and viral transduction of neurons (green) relative to the facial nucleus $(7 \mathrm{~N})$ and NMB+ RTN neurons (red). G) AAV9-SynGCaMP6s injection into the RVLM. H) GCaMP6s fluorescence of transduced RVLM neurons in freely behaving mice. I) Micrograph of lens placement and viral transduction of neurons (green) and PNMT+ RVLM neurons (red). Scalebar in panel F and I represent 200 $\mu \mathrm{m}$; Abreviations: Ad, adapting; NC, non-coding; In, inhibited; Gr, graded; 7N, facial motor nucleus; Py, pyramidal tract; MVe, medial vestibular nucleus; sp5, spinal trigeminal nucleus: RTN, retrotrapezoid nucleus; RMg, raphemagnus; RPa, raphe pallidus; ROb, raphe obscurus; pFL, parafacial lateral region; RVLM, rostral ventrolateral medulla; NA, nucleus ambiguus; NeuN, neuronal nuclei; GFAP, glial fibrillary acidic protein; NMB, neuromedin B; PNMT, phenylethanolamine N-methyltransferase. 

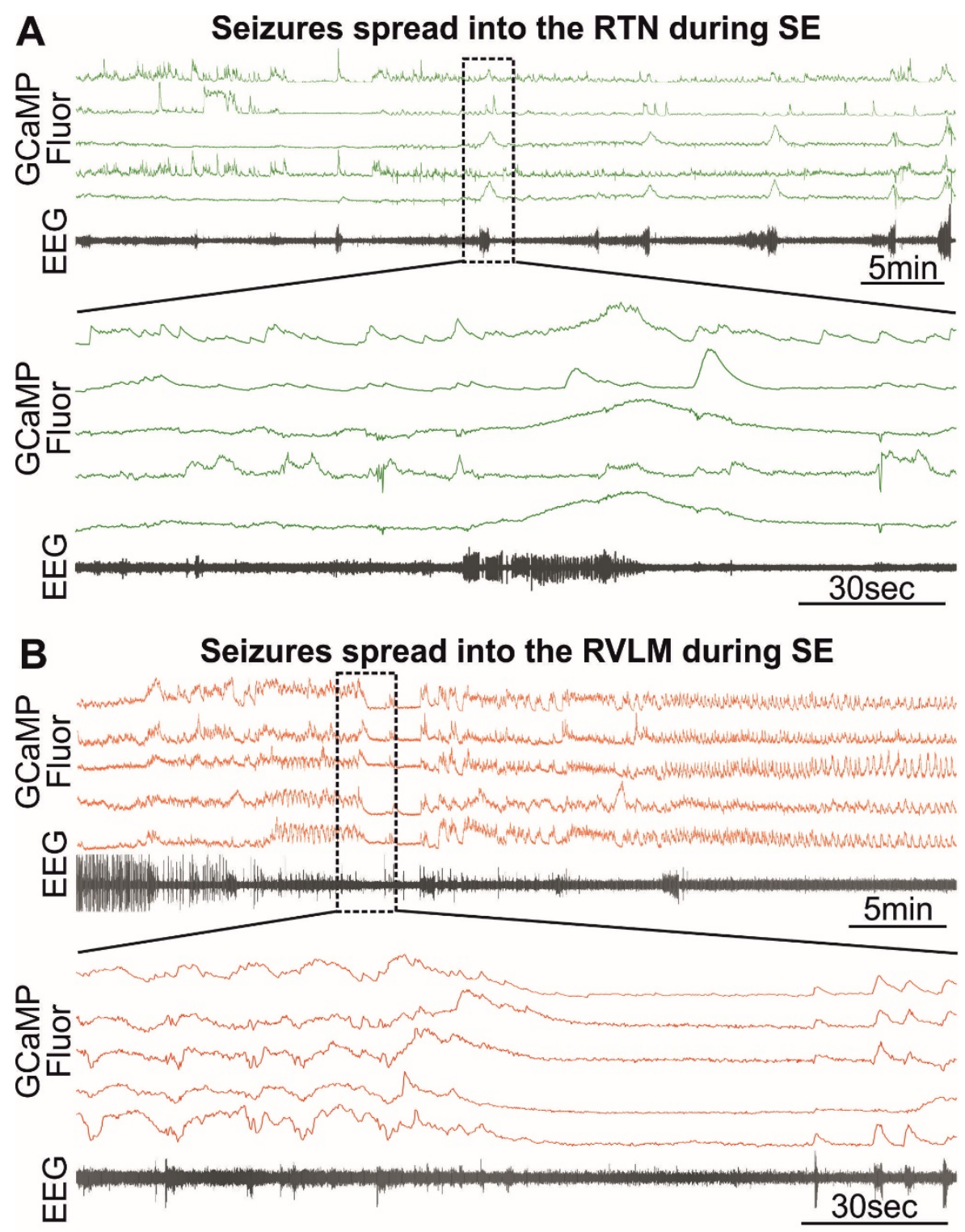

C

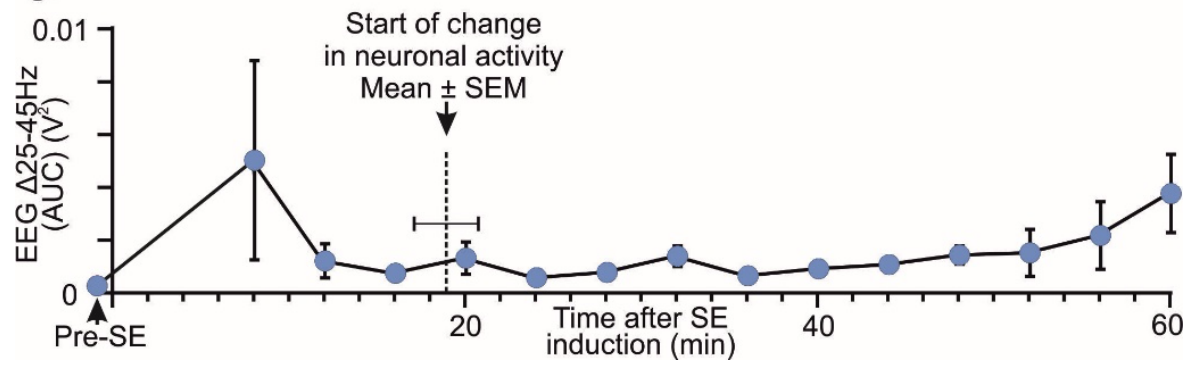

Figure 2: A) KA-induced seizures during status epilepsy (SE) spread into the RTN nuclei. The dotted square shows changes in activity of RTN neurons in freely behaving mice time matched with EEG seizure and expanded under it. Seizure activity after intrahippocampal injection of KA time matched with the activity of RTN neurons (green). B) KA-induced seizures during status epilepsy spread into the RVLM nuclei. The dotted square shows changes in activity of RVLM neurons in freely behaving mice time matched with EEG seizure and expanded under it. Seizure activity after intrahippocampal injection of KA time matched 
with the activity of RVLM neurons (red). C) Start of seizure activity i.e., changes in amplitude of gamma-range frequency $(25-45 \mathrm{~Hz})$ before induction of SE (pre-SE) and after intrahippocampal injection of $\mathrm{KA}(\mathrm{n}=8)$. Increase in the gamma range frequency $(25-45 \mathrm{~Hz})$ in EEG is characteristic property of KA-induced seizures ${ }^{11,12}$. The seizure start time is compared with the time of first visual aberrant cardiorespiratory neuronal activity calculated from respective $\mathrm{Ca}^{2+}$ traces from mice recorded for RTN and RVLM ( $\left.n=14\right)$. The start of change in neuronal activity is represented as a dotted line.
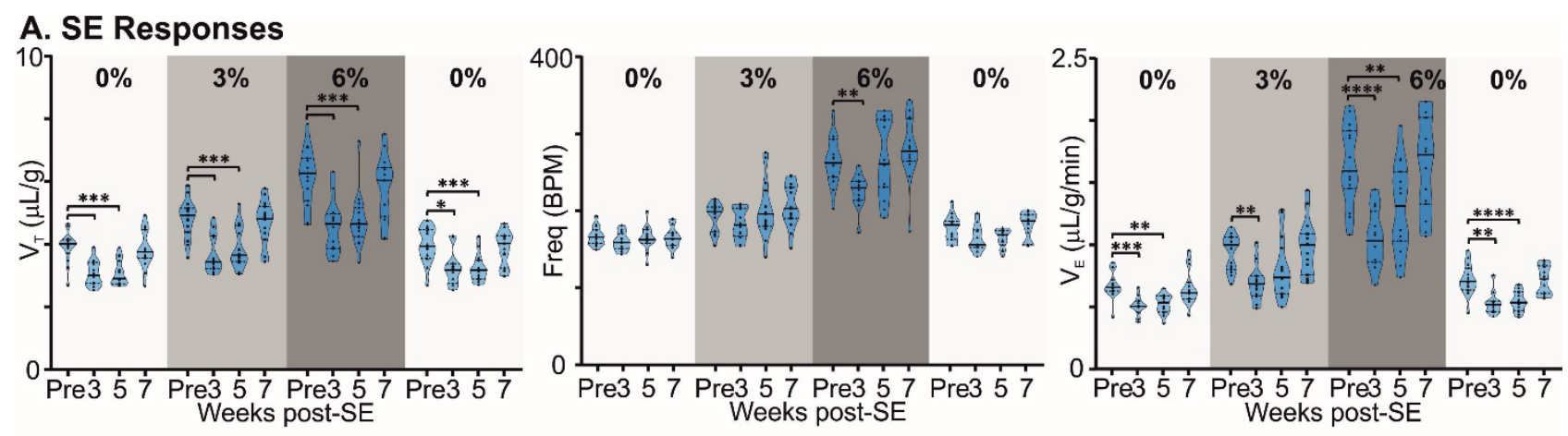

\section{B. Control Responses}
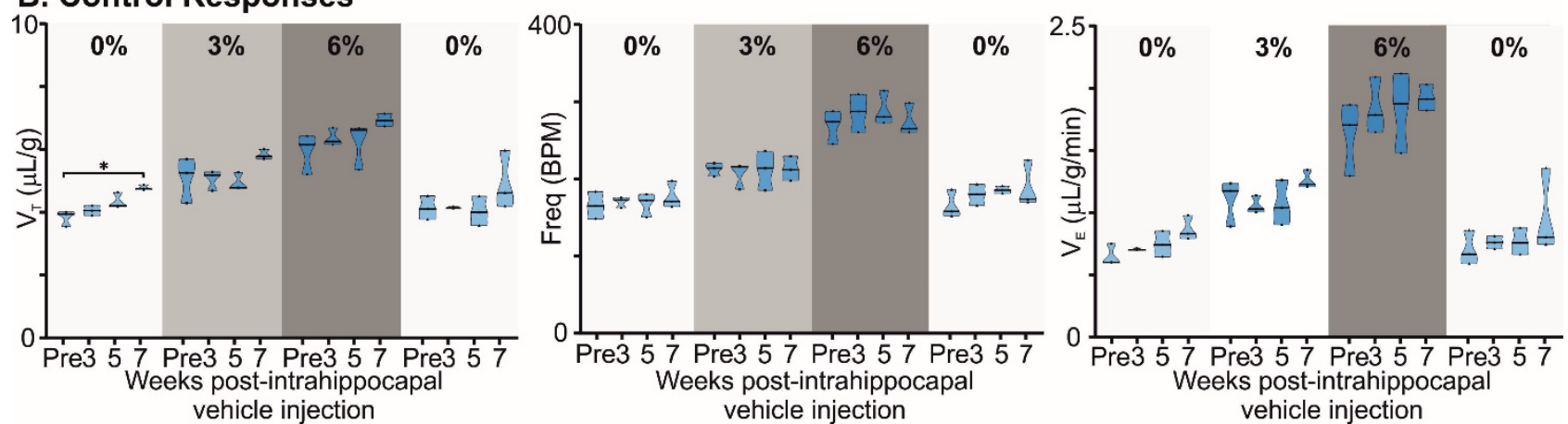

Figure 3: A) Changes in tidal $\left(V_{T}\right)$, frequency $\left(f_{R}\right)$ and minute ventilation $\left(V_{E}\right)$ in response to 3 and $6 \% \mathrm{CO}_{2}$ before and 3,5 and 7-weeks after induction of SE in freely behaving mice $(n=15)$. Median and quartile are shown with continuous and dotted line, respectively. B) Changes in tidal $\left(\mathrm{V}_{T}\right)$, frequency $\left(\mathrm{f}_{\mathrm{R}}\right)$ and minute ventilation $\left(\mathrm{V}_{\mathrm{E}}\right)$ in response to 3 and $6 \%$ $\mathrm{CO}_{2}$ before and 3, 5 and 7-weeks after injection of intrahippocampal PBS in freely behaving mice (control; $\mathrm{n}=3$ ). Median shown with continuous line. $\mathrm{P}$ values derived from two-way repeated measure ANOVA are ${ }^{*} p<0.05,{ }^{* *} p<0.001,{ }^{* * *} p<0.001$ and ${ }^{* * *} p<0.0001$. 


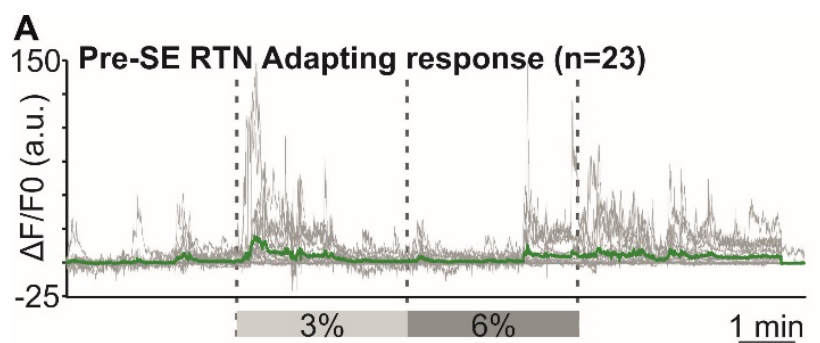

150 5 weeks Post-SE RTN Adapting response $(n=10)$

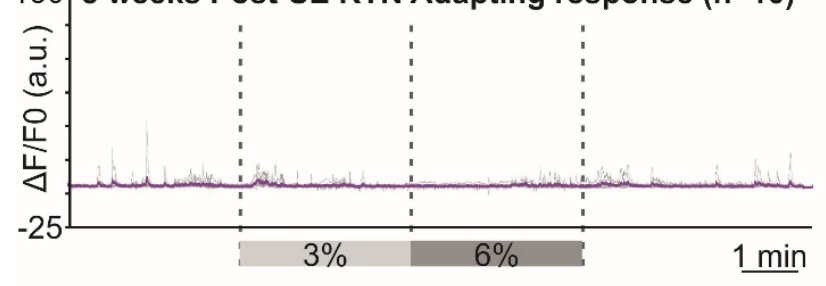

B 25 RTN Adapting responses

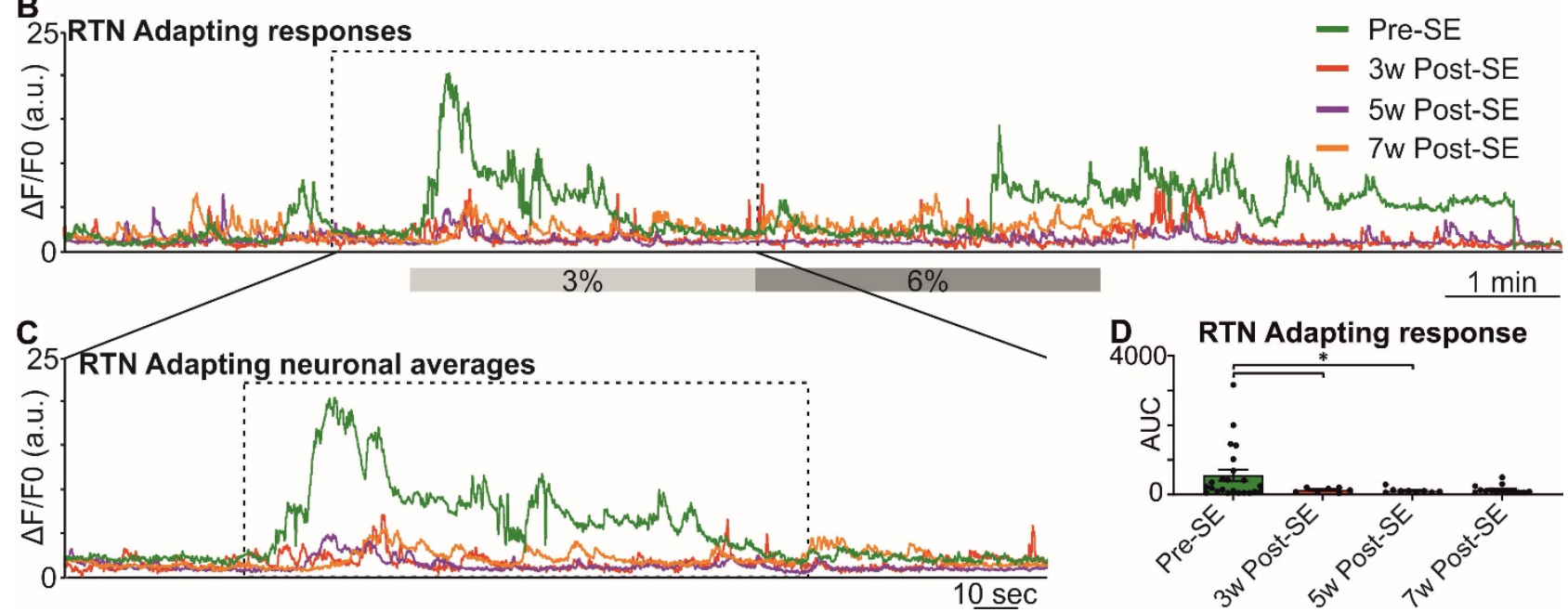

Figure 4: A) RTN neurons individual and average adapting responses to hypercapnia before and 3, 5 and 7 -weeks after induction of epilepsy in freely behaving mice. $n$ is number of neurons recorded from 3 mice. In 7-weeks post-SE panel, for technical reasons the recording of recovery from hypercapnia is absent. B) RTN neurons average adapting responses compared between pre and 3,5 and 7-weeks post-SE. The dotted square shows changes in adapting response of RTN neurons in freely behaving mice and expanded in panel C. C) Changes in RTN neurons adapting response to $3 \% \mathrm{CO}_{2}$ (dotted box) with prehypercapnic baseline activity. D) RTN neurons adapting response to $3 \% \mathrm{CO}_{2}$ (from the dotted box in panel C) measured as the area under the curve (AUC). Data are mean \pm SEM. $P$ values derived from Brown-Forsythe and Welch ANOVA is ${ }^{*} p<0.05$. 

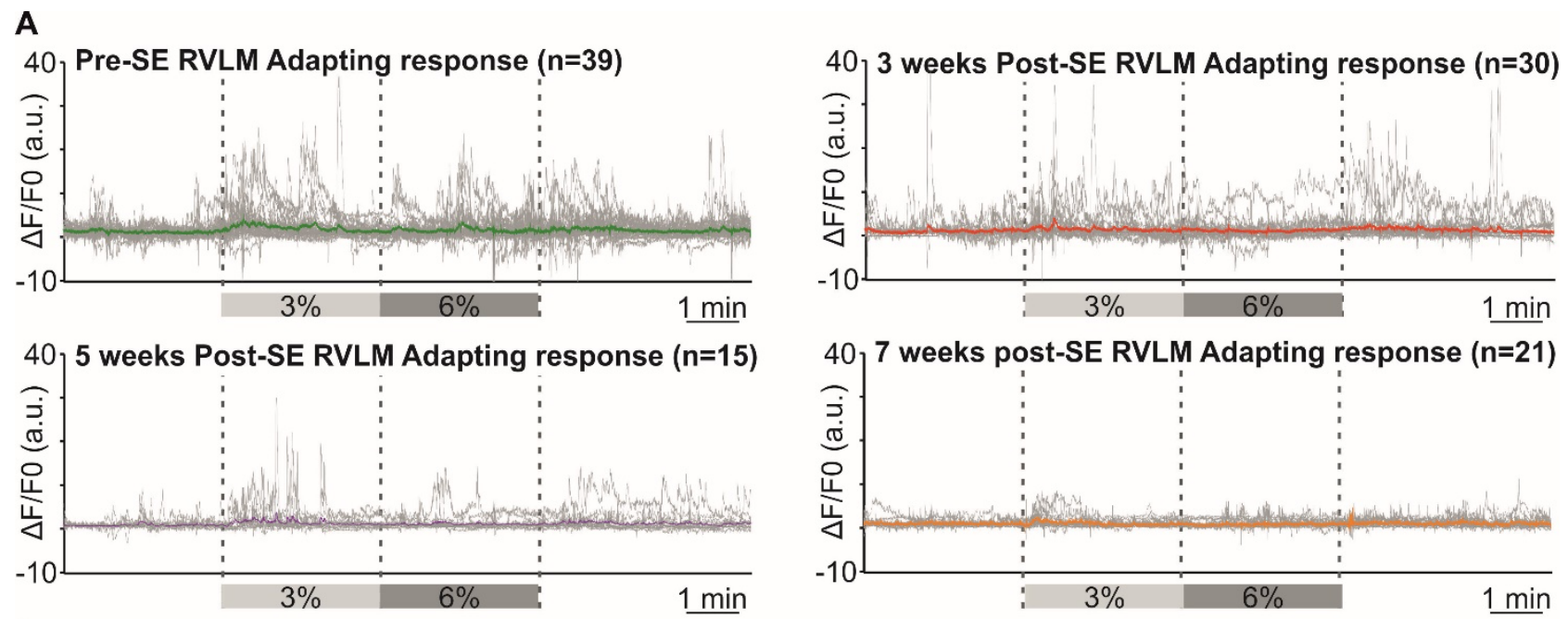

\section{${ }_{5}$ RVLM Adapting responses}

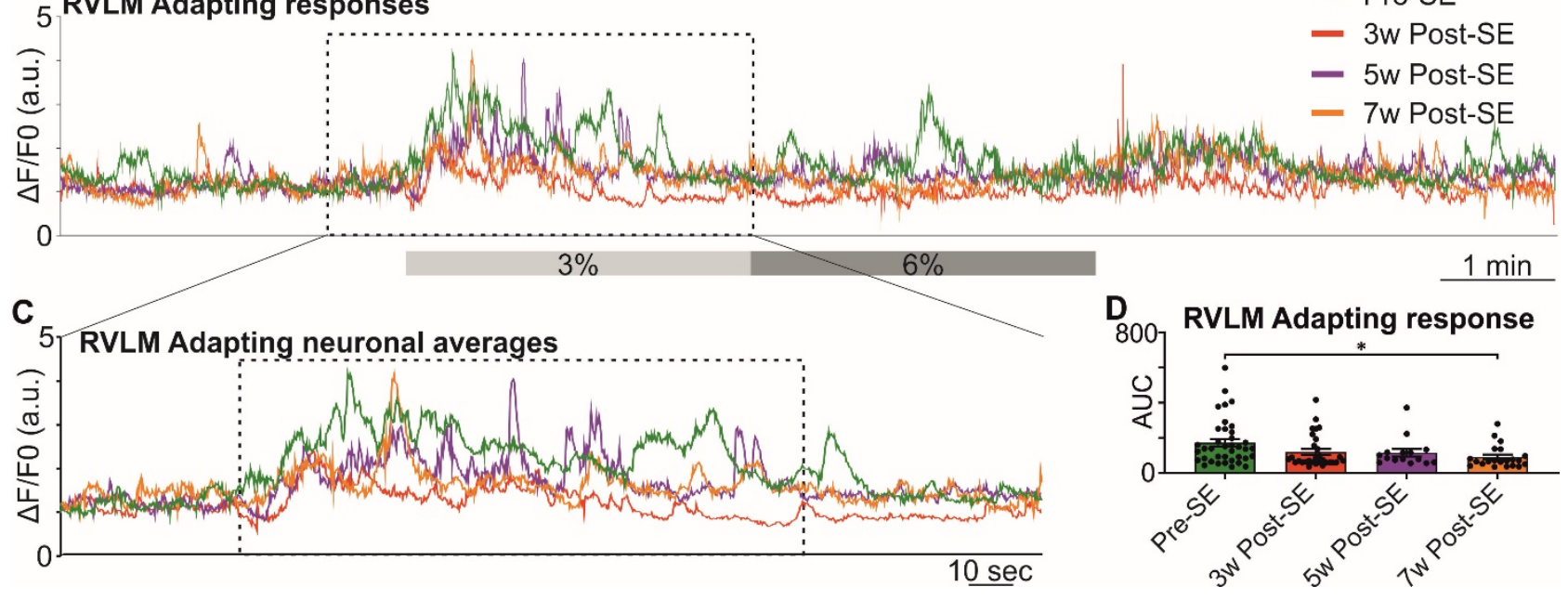

- Pre-SE

- 3w Post-SE

- 5w Post-SE

- 7w Post-SE

Figure 5: A) RVLM neurons individual and average adapting responses to hypercapnia before and 3, 5 and 7-weeks after induction of epilepsy in freely behaving mice. $n$ is number of neurons recorded from 7 mice. B) RVLM neurons average adapting responses compared between pre and 3, 5 and 7-weeks post-SE. The dotted square shows changes in adapting response of RVLM neurons in freely behaving mice and expanded in panel C. C) RVLM neurons adapting response to $3 \% \mathrm{CO}_{2}$ (dotted box) with pre-hypercapnic baseline activity. D) RVLM neurons adapting response to $3 \% \mathrm{CO}_{2}$ (from the dotted box in panel $\mathrm{C}$ ) measured as the AUC. Data are mean \pm SEM. $P$ value derived from one-way ANOVA is ${ }^{*} p<0.05$. 
A

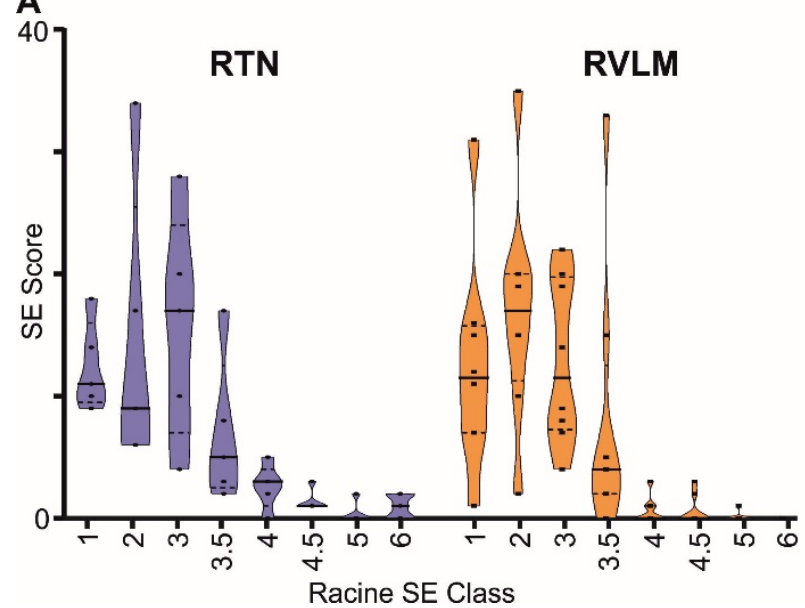

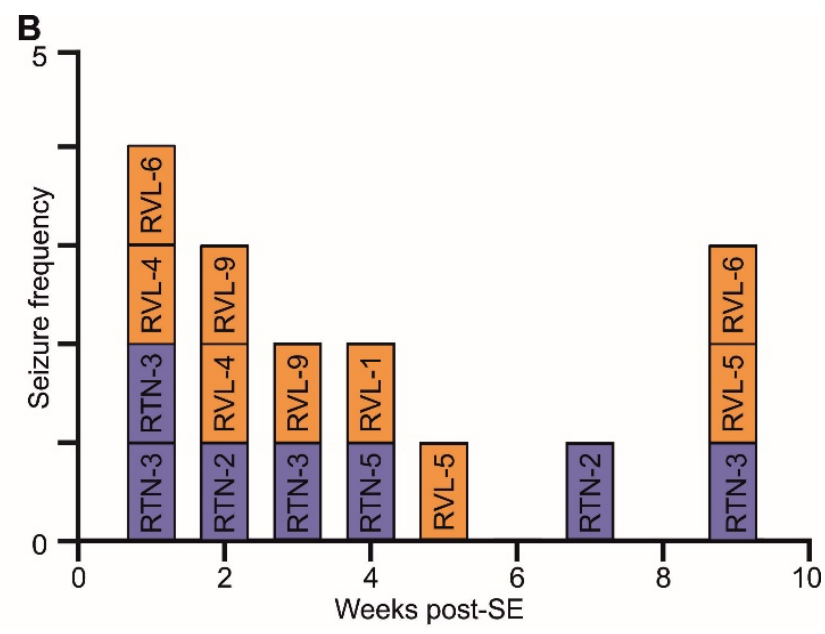

Figure 6: A) Comparison of Racine behavioural scale score during induction of SE in mice recorded for RTN and RVLM. x-axis, Racine behavioural class and y-axis, number (score) of specific SE behaviour. Median and quartile are shown with continuous and dotted line, respectively. B) Weekly spontaneous seizures frequency noted (during routine mouse monitoring), and video recorded in individual mice recorded for RTN and RVLM neuronal activity after induction of SE.
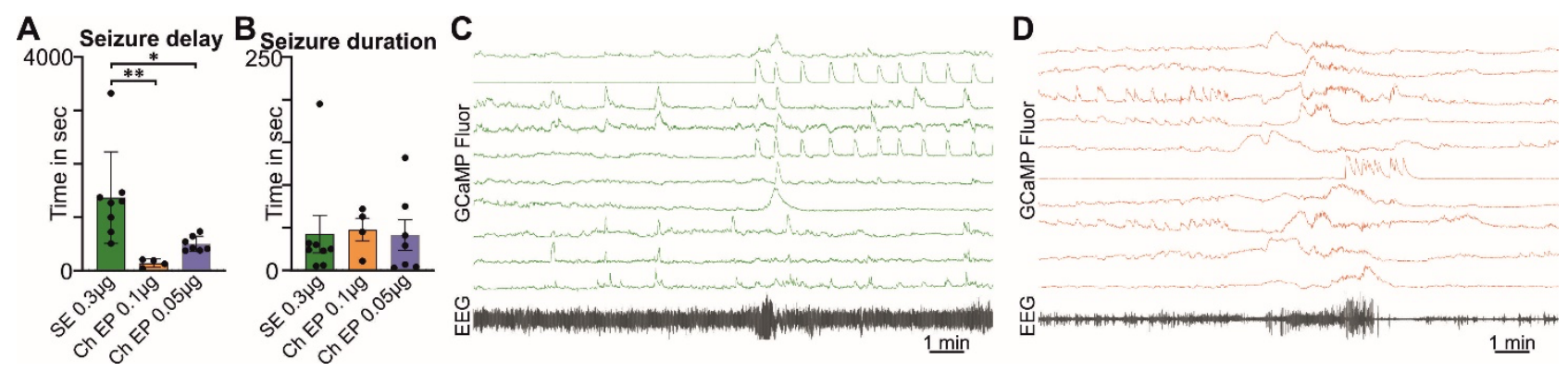

Figure 7: During chronic epilepsy in mice A) seizure delay and B) duration time with $3(n=4)$ and $6(n=7)$ fold lower dose of intrahippocampal KA is compared to SE $(n=8) . n=8,4$ and 7 for $0.3,0.1$ and $0.05 \mu \mathrm{g}$ of hippocampal KA injection. Data are mean \pm SEM. $P$ values derived from one-way ANOVA are ${ }^{*} p<0.05$ and ${ }^{* *} p<0.001$. Effect of 6-fold lower dose of intrahippocampal KA (compared with the SE) on C) the RTN and D) the RVLM neuronal activity with reduced seizure induction delay. 
Supplementary Movie 1: Seizures spread into the RTN neurons and disturb their activity during induction of SE via intrahippocampal KA injection in freely behaving mice. Movie is $8 \mathrm{x}$ fast forwarded.

Supplementary Movie 2: Seizures spread into the RVLM neurons and disturb their activity during induction of SE via intrahippocampal KA injection in freely behaving mice. Movie is $8 \mathrm{x}$ fast forwarded.

Supplementary Movie 3: During chronic epilepsy, 3 times of lower dose of KA (0.1 $\mu \mathrm{g})$ compared to SE showed reduced latency for induction and spread of seizures into the RVLM neurons. Movie is $8 x$ fast forwarded.

Supplementary Movie 4: During chronic epilepsy, 6 times of lower dose of KA (0.05 $\mu \mathrm{g})$ compared to SE showed reduced latency for induction and spread of seizures into the RTN neurons. Movie is $8 x$ fast forwarded. 